\title{
Evaluation of a screening chest X-ray programme for the detection of pulmonary tuberculosis in asymptomatic military members ${ }^{\dagger}$
}

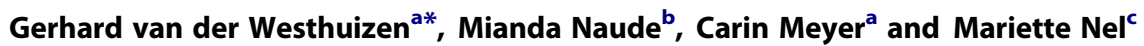 \\ ${ }^{a}$ Department of Clinical Imaging Sciences, University of the Free State, Bloemfontein, South Africa \\ ${ }^{b}$ Head of Department, Department of Radiology, 3 Military Hospital, Bloemfontein, South Africa \\ 'Department of Biostatistics, University of the Free State, Bloemfontein, South Africa \\ *Corresponding author, email: Gerhardvdwest@gmail.com
}

This study evaluated reports from screening chest X-rays (s-CXRs) done by the South African Military Health Services on asymptomatic military members over the age of 40 . Focus was placed on the yield of tuberculosis (TB) positive s-CXRs and a total of 29 (1.2\%) patients showed signs of active TB from a study population of 2371 patients during a 54-month study period. Although the results from this study cannot be extrapolated to the general population, the findings indicate that screening of the general population could be of value in South Africa where there is a high incidence of TB.

Keywords: asymptomatic, chest radiographs, chest X-rays, military, screening, tuberculosis

\section{Introduction}

A screening chest X-ray (s-CXR) is done on all South African National Defence Force members over the age of 40 as part of a health assessment. It is stipulated in The South African Military Health Service's Process and Procedures on Health Care Delivery that it should be done yearly on combat-ready members and every two years on non-combat-ready members to determine the member's functional and operational status by excluding pulmonary tuberculosis (PTB) and other significant pathology. Patients with a PTB positive s-CXR are then subjected to further tests to confirm or exclude active PTB.

The 2015 global tuberculosis report, released by the World Health Organization (WHO) in 2016, reported that South Africa had the sixth highest incidence of tuberculosis (TB) in the world with a total of 454000 new cases and the highest incidence rate with 834 new cases per 100000 population. ${ }^{1}$ A statistic to take note of was released by Statistics South Africa, which stated that the prevalence of TB amongst South African youth (14 to 35 years old) was $2.9 \%$ countrywide, and $2.1 \%$ in the Free State province in 2013. ${ }^{2}$ The prevalence of TB in the general population during that same year was $0.7 \%$ (715 per 100000$).^{3}$

Screening for TB is uncommon in developed countries with screening mainly reserved for immigrants originating from countries with a high incidence of PTB. A study by Alvarez et al. showed that most countries use s-CXRs for initial screening, whereafter sputum microscopy and culture or the Xpert MTB/RIF test (Cepheid, Sunnyvale, CA, USA) is used for confirmation of diagnosis. This study reported that the s-CXR is not the gold standard TB screening test, with sensitivity and specificity of diagnosing active PTB only in the range of $64-80 \%$ and $52-$ $63 \%$ respectively. This study, however, also found that radiographic screening demonstrated benefits in screening large numbers of individuals in high prevalence populations. ${ }^{4}$ More recent data were published in 2014 by TB CARE I stating a pooled sensitivity and specificity of $87-98 \%$ and $56-89 \%$ respectively of $\mathrm{s}-\mathrm{CXRs} .^{5} \mathrm{~A}$ high rate of inter- and intra-observer variability as well as an atypical radiographic pattern seen in patients with both pulmonary tuberculosis and HIV decreases the sensitivity and specificity of s-CXRs. ${ }^{6}$ The WHO TB screening guidelines, released in 2013, recommend that s-CXR should still be used as second-line screening tool (TB symptoms screening is the first-line screening tool), followed by the Xpert MTB/RIF test for confirmation of diagnosis. ${ }^{7}$ This is suggested when both sCXRs and the Xpert MTB/RIF test are available as screening tools, as is the case in our screening programme.

The largest study to evaluate the efficacy and cost-effectiveness of radiographic screening of a military population for PTB in a resource-limited country was published by Supramaniam in 1980. The Malaysian military was screened with mass miniature radiographs (MMAs) and only 112 (approximaxtely $0.1 \%$ ) cases of PTB were diagnosed from more than 100000 MMAs over a 10 -year period. It was concluded that radiographic screening for PTB was not cost-effective. ${ }^{7}$ The suggestion had already been made in 1974 by the WHO's Expert Committee on Tuberculosis that MMAs should be abandoned as a screening tool for active TB. ${ }^{8}$ The South African Military Health Services have replaced MMAs with standard posterior-anterior (PA) CXRs due to the high radiation dose and low image quality of MMAs. The radiation dose from a single PA CXR is $0.1 \mathrm{mSV}$, which is the equivalent of approximately 10 days' background radiation. ${ }^{9}$ The TB incidence in South Africa during our study period was approximately 12 times higher than the TB incidence in Malaysia in 1985 (oldest available data). ${ }^{1}$

Radiological diagnosis of active PTB in adults is usually indicative of reactivation/post-primary TB. The diagnosis is most commonly made when there is focal or patchy heterogeneous air space consolidation present in the apical and posterior segments of the upper lobes and the superior segments of the

\footnotetext{
${ }^{\dagger}$ Abstract presentation: The complete data from the study were presented at the RSSA/SASPI conference held at Spier, Stellenbosch from November 36, 2016 and the abstract was published in the South African Journal of Radiology on April 28, 2017 under the title: 'The Yield of Pathological Findings from Routine Screening Chest X-rays in a Military Population' (S Afr J Radiology. 2017;21(1):a1127. doi: 10.4102/sajr.v21i1.1127). The research presentation was awarded first prize in the travel award category. Only data pertaining to TB was used for this brief report.
} 
lower lobes. Cavitation is also highly suggestive of reactivation $T B$, especially when this finding is seen in conjunction with other signs of active PTB including pleural effusions (which may be the sole finding), tuberculomas, poorly defined nodules, bronchiectasis and lymphadenopathy. Cavitation becomes a less specific sign of active PTB when it is seen without these additional signs and when healing by fibrosis is already present (fibrocavitary changes). Residual fibrocavitary changes can be present even in the absence of active PTB.

The radiological findings of HIV-associated PTB are dependent on the level of immunosuppression. Patients with a CD4 T-lymphocyte count of less than $200 / \mathrm{mm}^{3}$ have a lower prevalence of cavitation and tend to present with prominent hilar and mediastinal lymphadenopathy, an atypical consolidation distribution or miliary disease. ${ }^{10}$

The aim of this study was to evaluate the efficacy of a s-CXR programme for the detection of PTB in asymptomatic military patients.

\section{Research method and design}

Approval for the study was obtained from the 1 Military Hospital Research Ethics Committee, as well as the Ethics Committee of the Faculty of Health Sciences of the University of the Free State.

A retrospective descriptive study was done on data of all military patients over the age of 40 on whom a s-CXR had been done at the Radiology Department of 3 Military Hospital between May 2011 and October 2015 as part of their health assessment. Routine s-CXRs are not done on members under the age of 40 and were thus excluded from the study. Symptomatic military members presented to the casualty unit or the sickbay and were excluded from the study. This ensured that any pathology identified on the s-CXRs was identified on asymptomatic military members.

Standard inspiratory PA CXRs were acquired at the time of the examination, using standard radiographic techniques. A radiologist or a radiology registrar working under consultant supervision evaluated and reported the s-CXRs. The retrospective nature of the study meant that the reporting doctors were unaware of the study at the time of reporting. The reports were stored on an electronic database, from which the data were retrieved and reviewed individually by the researcher. Only the final s-CXR reports were evaluated. The images were not retrospectively reviewed and the radiological findings included in the final reports were not double-checked. This was done intentionally, considering that these reports were used for clinical decision-making and further management of the patients.

Demographic data collected from the s-CXR reports included the patient's hospital number (for administrative purposes), age and gender.

A wide spectrum of data was collected from the s-CXR reports, but only the findings discussed in the introduction relating to either active PTB, possible PTB or previous PTB were included in this report.

Descriptive statistics, including frequencies and percentages for categorical data, and medians and percentiles for continuous data were calculated. The prevalence of significant findings was calculated and described by means of the $95 \%$ confidence interval for the prevalence. Statistical analysis was done by the Department of Biostatistics at the University of the Free State.

\section{Results}

This retrospective study included a total of 4137 examinations done on 2371 patients during the 54-month study period. Of these, 2114 (89.2\%) were male and 257 (10.8\%) were female. The median age of the patients at the time of first examination was 43.9 years (range 40.0-77.2). Of the total sample, 2062 (86.9\%) patients were between 40 and 49 years, $287(12.1 \%)$ between 50 and 60 years and only $22(0.9 \%)$ were older than 60 years at the time of examination.

Table 1 is a summary of all the positive findings identified on $\mathrm{s}-\mathrm{CXR}$ during the study period. The initial s-CXR pertains to the first s-CXR done on each of the 2371 patients during the study period and the results are given in the first results column. The second column includes the cumulative findings from all (initial and subsequent) s-CXRs done on the 2371 patients during the study period. The findings pertaining to PTB are indicated in bold. The rest of the findings included in the table were the most common findings included in the sCXR reports but will not be discussed further in this report.

A total of 29 (1.2\%) patients were diagnosed with active PTB on s-CXR during the study period ( $95 \% \mathrm{Cl}$ for the prevalence $0.9 \%-1.8 \%$ ). In

Table 1: Summary of findings on s-CXRs during the study period

\begin{tabular}{|c|c|c|}
\hline \multirow[b]{3}{*}{ Findings } & Initial s-CXR & All s-CXRs \\
\hline & $\begin{array}{c}\text { ( } n=2371 \\
\text { patients) }\end{array}$ & $\begin{array}{c}(n=2371 \\
\text { patients) }\end{array}$ \\
\hline & Frequency (\%) & Frequency (\%) \\
\hline \multicolumn{3}{|l|}{ Significant findings: } \\
\hline Pleural effusion & $29(1.2)$ & $36(1.5)$ \\
\hline Active pulmonary TB & $21(0.9)$ & $29(1.2)$ \\
\hline Lymphadenopathy & $22(0.9)$ & $29(1.2)$ \\
\hline Cardiomegaly & 39 (1.6) & $62(2.6)$ \\
\hline Hyperinflation & $40(1.7)$ & $64(2.7)$ \\
\hline $\begin{array}{l}\text { Pulmonary venous } \\
\text { hypertension }\end{array}$ & $30(1.3)$ & $41(1.7)$ \\
\hline Bronchopneumonia & $19(0.8)$ & $26(1.1)$ \\
\hline Lobar pneumonia & $13(0.6)$ & $23(1.0)$ \\
\hline Bronchiectasis & $9(0.4)$ & $12(0.5)$ \\
\hline Solitary pulmonary nodule & $5(0.2)$ & $9(0.4)$ \\
\hline Interstitial lung disease & $6(0.3)$ & $6(0.3)$ \\
\hline $\begin{array}{l}\text { Pulmonary arterial } \\
\text { hypertension }\end{array}$ & $2(0.1)$ & $3(0.1)$ \\
\hline Suspicious bone lesion & $0(0)$ & $1(0.0)$ \\
\hline \multicolumn{3}{|l|}{ Insignificant findings: } \\
\hline Fibrocavitary changes & $98(4.1)$ & $125(5.3)$ \\
\hline Granulomas & $46(1.9)$ & $62(2.6)$ \\
\hline Calcified lymph nodes & $2(0.1)$ & $8(0.3)$ \\
\hline Peribronchial cuffing & $60(2.5)$ & $88(3.7)$ \\
\hline Unfolded aorta & $32(1.4)$ & $59(2.5)$ \\
\hline Thoracic scoliosis & $24(1.0)$ & $35(1.5)$ \\
\hline Previous rib fracture & $23(0.9)$ & $31(1.3)$ \\
\hline Previous clavicle fracture & $12(0.5)$ & $19(0.8)$ \\
\hline Previous sternotomy & $7(0.3)$ & $8(0.3)$ \\
\hline Shrapnel & $7(0.3)$ & $7(0.3)$ \\
\hline Hiatus hernia & $4(0.2)$ & $4(0.2)$ \\
\hline Pacemaker & $2(0.1)$ & $2(0.1)$ \\
\hline Previous mastectomy & $2(0.1)$ & $2(0.1)$ \\
\hline
\end{tabular}


Table 2: Summary of all s-CXRs done on the 29 patients diagnosed with pulmonary tuberculosis during the study period

\begin{tabular}{|c|c|c|c|c|c|c|c|c|}
\hline Case no. & Age at initial & Gender & \multicolumn{5}{|c|}{ Year of s-CXR } & \multirow{2}{*}{$\frac{\text { Number of }}{\text { PTB }+ \text { s-CXRs }}$} \\
\hline & PTB + s-CXR & $M=26(89.7 \%)$ & 2011 & 2012 & 2013 & 2014 & 2015 & \\
\hline$(n=29)$ & (Median age: 43.9 years) & $F=3(10.3 \%)$ & & & & & & per patient \\
\hline 1 & 51.4 & M & PTB+ & PTB- & & & & 1 \\
\hline 2 & 41.7 & M & PTB- & PTB+ & PTB+ & & & 2 \\
\hline 3 & 49.4 & M & PTB + & PTB- & PTB- & PTB- & & 1 \\
\hline 4 & 43.9 & M & PTB+ & & & PTB + & & 2 \\
\hline 5 & 51.9 & M & PTB- & & PTB- & PTB- & PTB+ & 1 \\
\hline 6 & 40.9 & M & & PTB+ & & & & 1 \\
\hline 7 & 40.4 & M & & PTB + & & & & 1 \\
\hline 8 & 52.0 & M & & PTB+ & & & & 1 \\
\hline 9 & 45.1 & $\mathrm{~F}$ & & PTB+ & PTB- & & & 1 \\
\hline 10 & 51.8 & M & & PTB- & & PTB+ & & 1 \\
\hline 11 & 45.6 & $M$ & & PTB- & PTB- & PTB+ & & 1 \\
\hline 12 & 40.5 & M & & PTB + & PTB- & PTB- & & 1 \\
\hline 13 & 41.2 & $M$ & & PTB+ & PTB+ & PTB+ & PTB- & 3 \\
\hline 14 & 41.5 & $M$ & & PTB+ & PTB+ & & PTB- & 2 \\
\hline 15 & 46.7 & M & & PTB+ & PTB- & & PTB- & 1 \\
\hline 16 & 45.8 & M & & PTB+ & & PTB- & PTB+ & 2 \\
\hline 17 & 48.2 & M & & PTB- & & PTB+ & PTB- & 1 \\
\hline 18 & 41.7 & $M$ & & & PTB+ & & & 1 \\
\hline 19 & 43.3 & $M$ & & & PTB+ & PTB+ & & 2 \\
\hline 20 & 41.9 & $\mathrm{~F}$ & & & PTB+ & & PTB- & 1 \\
\hline 21 & 43.6 & $M$ & & & PTB- & PTB+ & PTB+ & 2 \\
\hline 22 & 54.1 & $M$ & & & PTB- & & PTB+ & 1 \\
\hline 23 & 49.8 & $M$ & & & & PTB+ & & 1 \\
\hline 24 & 44.1 & M & & & & PTB- & PTB+ & 1 \\
\hline 25 & 40.1 & $\mathrm{~F}$ & & & & PTB+ & PTB+ & 2 \\
\hline 26 & 43.8 & $M$ & & & & & PTB+ & 1 \\
\hline 27 & 44.1 & $M$ & & & & & PTB+ & 1 \\
\hline 28 & 43.6 & $M$ & & & & & PTB+ & 1 \\
\hline 29 & 43.5 & $M$ & & & & & PTB+ & 1 \\
\hline Number o & + s-CXRs per year & & 3 & 10 & 6 & 9 & 10 & 38 \\
\hline
\end{tabular}

PTB -: No signs of active pulmonary tuberculosis on s-CXR.

PTB +: Signs of active pulmonary tuberculosis on s-CXR.

total, 21 (0.9\%) patients were diagnosed with PTB based on the findings on initial s-CXR (95\% Cl for the prevalence $0.6 \%-1.4 \%), 6(0.3 \%)$ patients on second s-CXR, 1 (0.04\%) patient on third s-CXR and 1 $(0.04 \%)$ patient on fourth s-CXR during the study period.

An analysis of the s-CXRs done on the 29 patients diagnosed with PTB during the study period is given in Table 2 . The table includes the gender of these patients, the age of the patients at initial diagnosis and whether the s-CXR was PTB positive or PTB negative for the year of assessment. The empty spaces indicate that no s-CXR was done on the patient for that specific year. The group median age of 43.9 years (range 40.1-54.1) was based on the patients' age at the time of initial PTB diagnosis during the study period. In total, $26(89.7 \%)$ patients were male and $24(82.8 \%)$ patients were between 40 and 49 years old.

Deductions that can be made from Table 2:

- Twenty-one (72.4\%) patients were diagnosed with PTB on initial s-CXR during the study period (not necessarily the patient's first s-CXR). Patients' TB statuses were unknown prior to the study period.
- Eight (27.6\%) patients were diagnosed with PTB on subsequent s-CXRs. These patients either contracted TB after initial s-CXR or PTB was missed on initial sCXR.

- Nine (31.0\%) patients had only one s-CXR during the study period on which PTB was diagnosed. The outcomes of these patients are unknown.

- Seven (24.1\%) patients were diagnosed with PTB on more than one s-CXR during the study period indicating either non-response to treatment (possible multi-drug resistant [MDR] TB), poor compliance or residual radiological findings of active PTB on subsequent s-CXR. Some patients had up to three PTB positive $s$-CXRs before having a normal s-CXR or remained PTB positive on the last included s-CXR during the study period.

- Nine (31.0\%) patients were successfully treated with no signs of active PTB on follow-up s-CXR.

- One (3.4\%) patient showed recurrence of PTB on the patient's third s-CXR after the second s-CXR was normal. This may have been due to either relapse or reinfection. 
- PTB was diagnosed on $38(0.9 \%)$ of the 4137 s-CXRs included in the study $(95 \% \mathrm{Cl}$ for the prevalence $0.7 \%-$ $1.3 \%)$.

Furthermore, a total of 62 (2.6\%) patients had non-specific findings (lymphadenopathy, pleural effusions or a combination of both). A total of 172 (7.3\%) patients had signs of previous PTB (fibrocavitary changes, granulomas, calcified lymph nodes or a combination of these findings) on their s-CXRs. These patients needed additional imaging or laboratory tests for a final diagnosis. As mentioned in the introduction, fibrocavitary changes are a non-specific finding and active PTB could not be excluded in these patients. The progression of these patients on follow-up imaging was not evaluated due to the non-specificity of these findings.

The HIV statuses of the patients were not available.

\section{Discussion}

The incidence rate of PTB from this study was significantly higher compared with the study published by Supramaniam, ${ }^{7}$ but comparing the results from the two studies was difficult due to a number of factors. Differences between the studies include: different radiographic techniques, a larger study population in the Malaysian study and the significantly higher TB incidence rate in the South African general population.

Numerous studies were identified on the Medline database that focused on screening high-risk groups for PTB, but none were identified that evaluated the efficacy of standard PA CXRs for the purpose of screening asymptomatic military members.

The demographic data (median age, age distribution and gender) of the 29 patients diagnosed with PTB on s-CXRs were similar to the demographic data of the total study population with no specific demographic group showing a higher incidence rate within our study population.

The percentage of patients with PTB in our study would be higher if any additional cases of PTB were diagnosed from the 62 patients with non-specific findings or from the 172 patients with signs of previous PTB.

Although the results from this study cannot be extrapolated to the general population, the number of additional PTB-positive patients identified in this study population (asymptomatic military members), suggests that additional cases of PTB would be detected in the general population if they were to be screened for PTB. The high incidence rate of TB in patients younger than 40 years suggests that screening should be initiated at a younger age.

TB symptoms screening should be the initial screening tool, followed by s-CXR and confirmed with the Xpert MTB/RIF test, as recommended by the WHO. Screening the general population would lead to earlier detection of active PTB, earlier initiation of treatment, a reduced risk of poor disease outcomes by reducing morbidity and mortality, and a reduction in TB transmission by decreasing close contact exposure time to the TB bacilli.

\section{Limitations of the study}

This study focused on the s-CXR reports, which meant that clinical history, findings on clinical examination and results of additional tests were not available. Patients younger than 40 years of age were excluded. The HIV statuses of the patients were unavailable.

\section{Conclusion}

This study demonstrated that screening asymptomatic military members for PTB using s-CXRs detected an unexpectedly high number of additional cases of PTB. Although the results of this study cannot be extrapolated to the general population, the findings indicate that a screening programme could be of benefit in high TB incidence countries like South Africa.

\section{Competing interests}

The authors declare that they have no financial or personal relationship(s) that may have inappropriately influenced them in writing this article.

Disclosure statement - No potential conflict of interest was reported by the authors.

Authors' contributions - G.v.d.W. was project leader, wrote the protocol, collected the data and wrote the manuscript. Mi.N. and C.M were study co-supervisors, assisted with planning of the study protocol and gave input on the final manuscript. Ma.N. assisted with the study protocol, analysed the data and gave input on the final article.

\section{References}

1. World Health Organization. Global tuberculosis report. Geneva: World Health Organization; 2016. Available from: http://apps.who. int/iris/bitstream/10665/250441/1/9789241565394-eng.pdf?ua=1

2. Statistics South Africa. Morbidity and mortality levels among the youth of South Africa. Pretoria: Statistics South Africa; 2013. Available from: http://www.statssa.gov.za/publications/Report-0309-12/Report-03-09-122013.pdf

3. Statistics South Africa. Millennium development goals 6: combat HIV/ AIDS, malaria and other diseases. Pretoria: Statistics South Africa; 2015. Available from: http://www.statssa.gov.za/MDG/MDG_Goal6_ report_2015_.pdf

4. Alvarez G, Gushulak B, Rumman K, et al. A comparative examination of tuberculosis immigration medical screening programs from selected countries with high immigration and low tuberculosis incidence rates. BMC Infect Dis. 2011;11(1):1-12. Available from: http:// www.ncbi.nlm.nih.gov/pmc/articles/PMC3022715/

5. TB CARE I. International Standards for Tuberculosis Care, Edition 3. TB CARE I, The Hague, 2014. Available from: http://www.currytbcenter. ucsf.edu/sites/default/files/istc_3rded.pdf

6. Koppaka R, Bock N. How reliable is chest radiography? In: Frieden T, editor. Toman's tuberculosis case detection, treatment, and monitoring: questions and answers. 2nd ed. Geneva: World Health Organization; 2004. Available from: http://apps.who.int/iris/ bitstream/10665/42701/1/9241546034.pdf

7. Supramaniam V. Is chest $x$-ray screening for pulmonary tuberculosis by mass radiography a cost-effective tool in a military population? Med J Malaysia [Internet]. 1980-Mar:34(3):301-6. Available from: http://www.ncbi.nlm.nih.gov/pubmed/6774221

8. World Health Organization. Systematic screening for active tuberculosis: principles and recommendations. Geneva: World Health Organization; 2013. Available from: https://www.ncbi.nlm.nih.gov/ books/NBK294081/

9. Mettler FA, Huda W, Yoshizumi TT, et al. Effective doses in radiology and diagnostic nuclear medicine: a catalog. Radiol. 2008;248:254-63. Available from: http://pubs.rsna.org/doi/pdf/10.1148/radiol. 2481071451

10. Jeong YJ, Lee KS. Pulmonary tuberculosis: up-to-date imaging and management. Am J Roentgenol. 2008;191:834-44. Available from: http://www.ajronline.org/doi/full/10.2214/AJR.07.3896 